ALZHEIMER DISEASE

\section{BACE1 inhibition could block CSF tau increase}

According to a new study in mice that exhibit amyloid pathology similar to that seen in Alzheimer disease (AD), inhibition of beta-site amyloid precursor protein-cleavage enzyme 1 (BACE1) can block amyloid deposition and prevent increases in the level of cerebrospinal fluid (CSF) tau. "Our findings demonstrate that CSF tau levels reflect amyloid- $\beta(\mathrm{A} \beta)$ deposits in the brain," summarizes Stephan Kaeser, who co-led the study.

Although fluid biomarker alterations in $\mathrm{AD}$ have been extensively examined, the underlying mechanisms of changing $A \beta$ and tau levels in the CSF over the disease progression remain incompletely

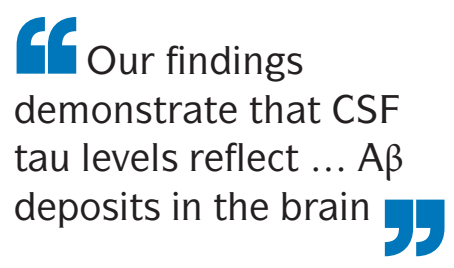

understood. Kaeser and colleagues set out to investigate the CSF biomarker changes in response to BACE1 inhibition, which is one of the leading strategies for early intervention in $\mathrm{AD}$.

The researchers fed NB-360, a potent BACE1 inhibitor, to two transgenic mouse strains, both of which have genetic alterations to amyloid precursor protein (APP), and show $\mathrm{A} \beta$ deposition with ageing. The researchers also developed a novel high-sensitivity sandwich assay for murine tau, using the single molecule array technology, which enables quantification of single molecules. "The improved sensitivity of our novel tau assay also allows a higher dilution of biological samples, which in turn helps to eliminate matrix effects," says Kaeser. "Our assay could potentially be used for human CSF samples, too, which is crucial for translational research."
As expected, treatment with BACE1 inhibitor reduced levels of CSF $A \beta$, and attenuated amyloid deposition in the brains of APP mutant mice. Moreover, BACE1 inhibition stabilized CSF tau levels, suggesting downstream effects on AD pathology.

According to Kaeser, CSF A $\beta_{42}$ levels may be a misleading readout for brain $A \beta$ deposition in the context of BACE1 inhibition because they are affected by two opposite mechanisms: BACE1 inhibition directly reduces the generation of $A \beta$ and thus lowers CSF A $\beta$ levels, but also attenuates $A \beta$ deposition that in turn leads to reduced $A \beta$ sequestration to amyloid plaques, which maintains elevated levels of CSF A $\beta_{42}$.

"The findings provide evidence that CSF tau is a reliable parameter to monitor the progression of $\mathrm{A} \beta$ pathology," says Kaeser. In future, CSF tau might become a pivotal biomarker for the efficacy assessment of BACE1 inhibitors in clinical trials.

Hemi Malkki

ORIGINAL ARTICLE Schelle, J. et al. Prevention of tau increase in cerebrospinal fluid of APP transgenic mice suggests downstream effect of BACE1 inhibition. Alzheimers \& Dementia http:// dx.doi.org/10.1016/j.jalz.2016.09.005 (2016) 